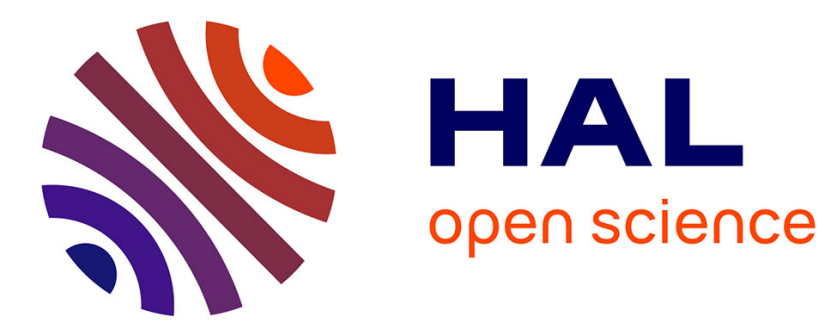

\title{
Closed-loop tool face control with the bit off-bottom
} Jean Auriol, Roman Shor, Ulf Jakob F. Aarsnes, Florent Di Meglio

\section{To cite this version:}

Jean Auriol, Roman Shor, Ulf Jakob F. Aarsnes, Florent Di Meglio. Closed-loop tool face control with the bit off-bottom. Journal of Process Control, 2020, 90, pp.35-45. 10.1016/j.jprocont.2020.03.011 . hal-02509642

\section{HAL Id: hal-02509642 \\ https://hal-centralesupelec.archives-ouvertes.fr/hal-02509642}

Submitted on 17 Mar 2020

HAL is a multi-disciplinary open access archive for the deposit and dissemination of scientific research documents, whether they are published or not. The documents may come from teaching and research institutions in France or abroad, or from public or private research centers.
L'archive ouverte pluridisciplinaire HAL, est destinée au dépôt et à la diffusion de documents scientifiques de niveau recherche, publiés ou non, émanant des établissements d'enseignement et de recherche français ou étrangers, des laboratoires publics ou privés. 


\title{
Closed-loop tool face control with the bit off-bottom
}

\author{
Jean Auriol ${ }^{\mathrm{a}, \mathrm{b}, *}$, Roman J. Shor ${ }^{\mathrm{a}}$, Ulf Jakob F. Aarsnes ${ }^{\mathrm{c}}$, Florent Di Meglio ${ }^{\mathrm{d}}$ \\ ${ }^{a}$ University of Calgary, Department of Chemical and Petroleum Engineering, Calgary, Canada \\ ${ }^{b}$ Université Paris-Saclay, CNRS, CentraleSupélec, Laboratoire des Signaux et Systìes, 91190, Gif-sur- Yvette, France \\ ${ }^{c}$ NORCE Norwegian Research Centre AS, Oslo, Norway \\ ${ }^{d}$ Centre Automatique et Systemes, Mines ParisTech, Paris, France
}

\begin{abstract}
In this paper, we develop three methods to achieve reliable closed-loop, tool face control for directional drilling operations. This is a necessary step to achieve closed-loop, automated directional guidance. Our algorithms combine existing industry top-drive controllers with new control approaches. The torsional model we use for the drill string has been field validated and takes into account the Coulomb friction between the drill string and the borehole. These distributed friction terms are either assumed known (or measured) or can be estimated using a state-observer. In this work, we improve such a state-observer to obtain an estimation of the tool face orientation in real-time. We then propose different approaches to control the tool face. The first method is based on a feed-forward control law. It uses the flatness of the model and the estimation of the orientation to generate an admissible trajectory which is then tracked. In the second procedure, we require a stable rotation off-bottom before smoothly changing the reference to zero to stop bit rotation. This change of reference induces a change of orientation that can be estimated and finally compensated by repeating the procedure. Finally, the last method uses a series of trapezoidal setpoint inputs - bumps - to calculate the change in downhole tool face per change in surface orientation before arriving at the correct tool face after three iterations. These three algorithms are illustrated in simulations of field scenarios and their effectiveness and limitations, depending on the reliability and availability of downhole orientation data, are discussed.
\end{abstract}

\section{Introduction}

To access resources in the subsurface, a slender borehole, between 10 and 60 centimeters in diameter, must be drilled along a precise wellpath from the surface to the subsurface target. To achieve precise positional control, two general classes of methods are typically used. In the first class, an active tool is placed near the drill bit which actively deflects the drill string to achieve directional capability, such as [1] or [2]. These tools usually have embedded closed loop feedback control systems and deliver smooth and precise wellpaths. However, these tools are often prohibitively expensive for low margin or low cost operations, are not capable of delivering high turn rates and are prone to failure but remain in development [3]. In the second class, a bent downhole mud motor is used. By placing a 1 to 5 degree bend in the pipe above a positive displacement motor (PWM) motor, steering may be achieved by alternatively rotating the bend - for a predominantly straight borehole - or holding the bend stationary and drilling soley using the downhole motor - for the curving wellbore. For this technique, a precise angular position - the tool face - of the bend is necessary to achieve directional guidance. Today, tool face is set by a human directional driller based

\footnotetext{
${ }^{*}$ Corresponding Author

Email addresses: jean.auriol@centralesupelec.fr (Jean Auriol)
}

on experience and limited downhole data which is communicated from downhole using either mud pulse telemetry or electromagnetic communication. Both of which are low bandwidth and high latency. Automated tool face control has been attempted, however, the long latency (1 to 20 seconds) of downhole telemetry has made automatic feedback control significantly slower than manual control $[4,5]$. Target zones for current wells are often thin (5 to 30 meters thick) layers of reservoir or source rock for hydrocarbons and precise placement of wells is critical for economic production of the insitu hydrocarbon. Typical directional wells have between 50 and 150 'slides' - where tool face is kept constant - for directional guidance, and human directional drillers spend up to 10 minutes setting tool face on each slide. Tool face accuracy may be as low as $\pm 45^{\circ}$, which results in highly tortuous wellbores which not only reduce drilling performance, but also future hydrocarbon production [6]. In this paper we propose three methods that achieve automatic closed loop tool face control with the drill bit (cutting tool located at the extremity of the drill string) off bottom. These methods enable faster and more precise geosteering in directional drilling.

\section{Model}

This section provides a recap of the torsional drill string model, with distributed friction terms, which is given in 
Table 1: Nomenclature

\section{Parameters}

$c_{t} \quad$ torsional wave velocity

$\mathcal{F} \quad$ Coulomb component of side force

$G, G_{p}, G_{c} \quad$ drill string, pipe, collar shear modulus

$I_{T D} \quad$ top drive inertia

$J, J_{p}, J_{c} \quad$ drill string, pipe, collar moment of inertia

$k_{t} \quad$ viscous component of side force

$L, L_{\underline{p}}, L_{c} \quad$ drill string, pipe, collar length

$\bar{Z} \quad$ collar-pipe relative impedance

$\zeta_{p} \quad$ pipe characteristic impedance

$\mu_{k}, \mu_{s} \quad$ kinetic, static friction coefficient

$\rho, \rho_{p}, \rho_{c} \quad$ drill string, pipe, collar density

$\omega_{c} \quad$ angular velocity threshold

$p_{\alpha}(\cdot), p_{\beta}(\cdot), p_{0}, p_{1}, P_{0}, P_{1}, l_{s}$ and $l_{k}$ are observer gains.

\section{Dependent variables}

$F_{N} \quad$ normal force on drill string

$\theta, \sigma_{e}$ angular and tension profile of the well

$W_{b} \quad$ buoyed weight per meter

$S$ torque source term (side force)

$\alpha, \beta \quad$ drill string Riemann invariants

$\omega \quad$ drill string angular velocity

$\tau \quad$ drill string torque

$\tau_{m} \quad$ motor torque

$\phi \quad$ tool face orientation

$\omega_{0} \quad$ Top drive angular velocity

$r_{o} \quad$ outer drill string radius

\section{Independent variables}

\section{$t$ time}

$x \quad$ position relative to top of drill string

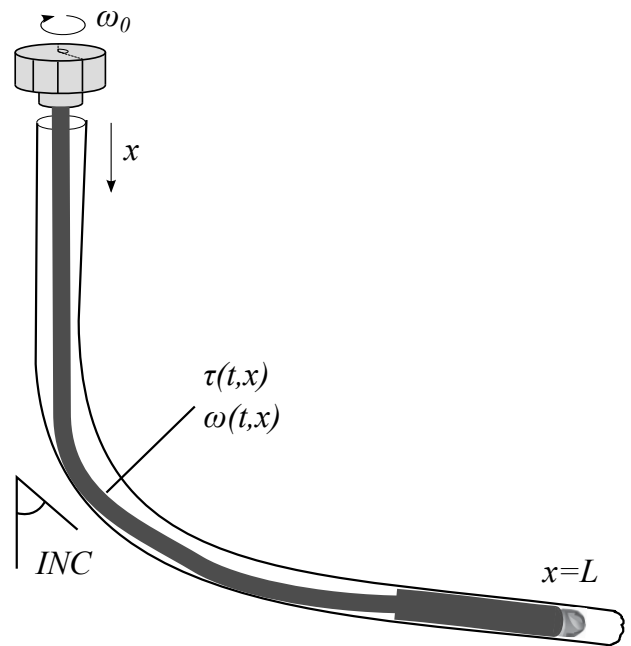

Figure 1: Schematic indicating the distributed drill string lying in deviate borehole.

full in [7]. The high fidelity - shown previously through comparisons with field data - and computational simplicity allows the model to be used in control and estimation applications. The main assumptions are as follows:

- Torsional motion is the dominant dynamic.

- Static and dynamic friction is modeled as a jump, i.e., the Stribeck curve is assumed negligible.

- The effect of along-string cuttings distribution is assumed constant and homogeneous.

- The effect of the pressure differential, inside and outside the drill string, on the bending moment is not represented and is assumed to be negligible.

\subsection{Torsional dynamics of drill string}

The torsional motion of the drill string is assumed to be the dominating dynamic behavior. The torsional dynamics is represented using the popular model (see [8, 9]) of a distributed wave model where discontinuities in impedance can be included to model different sections of the drill string, such as a pipe and a collar section. We refer to [7] for the full model derivation.

A schematic representation of the drill string is given in Fig. 1. We denote the angular velocity and torque as $\omega(t, x), \tau(t, x)$, respectively, with $(t, x) \in[0, \infty) \times[0, L](L$ being the length of the drill string). We have

$$
\begin{aligned}
& \frac{\partial \tau(t, x)}{\partial t}+J G \frac{\partial \omega(t, x)}{\partial x}=0 \\
& J \rho \frac{\partial \omega(t, x)}{\partial t}+\frac{\partial \tau(t, x)}{\partial x}=S(t, x),
\end{aligned}
$$

where, $J$ is the polar moment of inertia, $G$ the shear modulus and $\rho$ the drill string density. The source term $S$ is modeled as

$$
S(t, x)=-k_{t} \rho J \omega(t, x)-\mathcal{F}(t, x),
$$




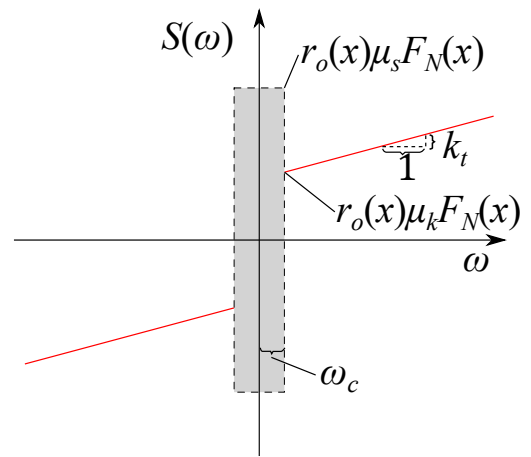

Figure 2: Schematic illustrating the friction source term $S(\omega, x)$ (as $S$ can be expressed as a function of $\omega$ ). The shaded region represents the angular velocities for which a constant value of static torque is assumed and the red curve indicates the dynamic torque as a function of angular velocity.

where the damping constant $k_{t}$ represents the viscous shear stresses and where $\mathcal{F}(t, x)$ is a differential inclusion that represents the Coulomb friction between the drill string and the borehole, also known as the side force. This side force is modeled using the following inclusion

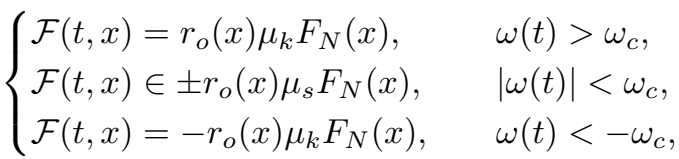

where $\mu_{s}$ is the static friction coefficient (i.e. the friction between two or more solid objects that are not moving relative to each other) and $\mu_{k}$ kinetic friction coefficient (also known as dynamic friction or sliding friction, which occurs when two objects are moving relative to each other and rub together), $\omega_{c}$ is the threshold on the angular velocity where the Coulomb friction transits from static to dynamic, $r_{o}(x)$ is the outer drill string radius. The function $F_{N}$ is the normal force acting between the drill string and the borehole wall. The function $\mathcal{F}(t, x) \in \pm r_{o}(x) \mu_{s} F_{N}(x)$ denotes the inclusion where

$$
\begin{aligned}
\mathcal{F}(t, x) & =-\frac{\partial \tau(t, x)}{\partial x}-k_{t} \rho J \omega(t, x) \\
& \in\left[-r_{o}(x) \mu_{s} F_{N}(x), r_{o}(x) \mu_{s} F_{N}(x)\right],
\end{aligned}
$$

and take the boundary values $\pm \mu_{s} F_{N}(x)$ if this relation does not hold. The shape of the friction source term is illustrated in Fig. 2. Using the torque model of [10] it is possible to derive the normal force profile $F_{N}(x)$. Assuming a planar well and torsional rotation of the drill string the normal force in terms of the tension profile $\sigma_{e}$ writes:

$$
\sigma_{e}(x)=\int_{L}^{x} W_{b}(\xi) \cos \theta \mathrm{d} \xi,
$$

where $W_{b}(x)$ is the buoyed weight per meter and $\theta$ is the angular profile. The normal force profile, $F_{N}$, is obtained as

$$
F_{N}(x)=\left(\sigma_{e}(x) \frac{\partial \theta}{\partial x}+W_{b}(x) \sin (\theta)\right)
$$

\subsection{Discontinuities of a multiple sectioned drill string}

The lower part of the drill string is usually made up of drill collars that may have a great impact on the global dynamics due to their inertia [11]. Due to the change of the characteristic line impedance, the transition from the pipes to collars in the drill string will cause reflections in the traveling waves. We split the drill string into a pipe section and a collar section. The corresponding inertia, length, density and shear modulus are respectively denoted $J_{p}, L_{p}, \rho_{p}, G_{p}$ and $J_{c}, L_{c}, \rho_{c}, G_{c}$. We use $\tau^{+}, \omega^{+}$ to denote the strain and velocity at the top of the drill collar and $\tau^{-}, \omega^{-}$at the bottom of the pipe. The boundary conditions at the transition are given by the following continuity constraints

$$
\omega^{+}=\omega^{-}, \quad \tau^{+}=\tau^{-} .
$$

\subsection{Top-drive boundary condition}

The top drive at the topside boundary is actuated by a motor torque, $\tau_{m}$, that is in most cases controlled using a PI control law [12] to reach a desired velocity set point $\omega_{S P}$ :

$$
\begin{aligned}
e & =\omega_{S P}-\omega_{T D}, \\
I_{e} & =\int_{0}^{t} e(\xi) \mathrm{d} \xi \\
\tau_{m} & =k_{p} e+k_{i} I_{e},
\end{aligned}
$$

where $k_{p}$ is a proportional gain and $k_{i}$ an integral gain. We denote $I_{T D}$ the topdrive inertia. We have the following equation

$$
\frac{d \omega_{T D}}{d t}=\frac{1}{I_{T D}}\left(\tau_{m}-\tau(t, 0)\right)
$$

In what follows, we denote $\omega_{0}=\omega(t, 0)$, the angular velocity at the top of the drill string. It verifies $\omega_{0}=\omega_{T D}$.

\subsection{Riemann invariants}

The Riemann invariants of a Hyperbolic PDE are the states that correspond to a transformation of the system for which the transport matrix has been diagonalized. With such a transformation, it becomes possible to write the system as a series of transport equations that are coupled through the source terms [13]. They are defined by

$$
\alpha_{i}=\omega_{i}+\frac{\left(c_{t}\right)_{i}}{J_{i} G_{i}} \tau, \quad \beta_{i}=\omega_{i}-\frac{\left(c_{t}\right)_{i}}{J_{i} G_{i}} \tau
$$

where $\left(c_{t}\right)_{i}=\sqrt{\frac{\rho_{i}}{J_{i}}}$ is the velocity of the torsional wave and where the index $i=c$ if we consider the collar section and $i=p$ if we consider the pipe section. The full derivation and an analysis of the effectiveness of this modeling approach has been explored in some details in [7]. 


\section{Estimation of the tool face orientation}

The model referenced in the previous section has been used to estimate downhole and along-string angular velocity and torque and has been validated with field data [14]. In this section, we extend this work to estimate in real time the tool face orientation. The tool face orientation is denoted by $\phi_{b}(t)$ and is defined by

$$
\phi_{b}(t)=\int_{0}^{t} \omega(\nu, L) d \nu+\phi_{0},
$$

where $\phi_{0}$ corresponds to the initial tool face orientation. More precisely, the observer designed in [14] combines the proposed model of the system dynamics with measurements from physical sensors. This observer relies on the measured top-drive angular velocity $\omega_{0}$. To be able to estimate the downhole orientation (which is the integral of the velocity), we also require downhole measurements, which may be sparse or latent.

During directional drilling operations, downhole measurements of the tool face orientation are transmitted to surface infrequently (on the order of once per minute to once per hour) and with considerable delay (on the order of seconds). Typical downhole sensors contain one to three axis accelerometers or gyroscopes and one to three axis magnetometers. These sensors are sampled at frequencies between 1-100 Hertz by the downhole tool, but only averaged or windowed values are transmitted to surface [15]. For human-in-the-loop operations, this is found to be sufficient since orienting the tool face takes between one and fifteen minutes. However, for automated solutions, in particular feedback controllers, this leads to significant performance degradation. Thus, the ability to estimate tool face in realtime provides the potential to significantly improve automated tool face orientation operations.

In our study we will assume a sampling rate of the tool face orientation measurements of 0.1 Hertz (i.e. every $T_{T}=10 \mathrm{~s}$ ), and with a delay of $\tau_{T}=10 \mathrm{~s}$, which is representative on a typical onshore horizontal well drilling operation. Consequently, these delayed tool face measurements cannot be used directly to update the observer estimates but must be compared to the observer estimate from the time the measurement was actually taken, based on which error the state estimate can be updated.

The estimation of the tool face orientation is obtained improving the soft-sensor introduced in [14] and based on the backstepping methodology [16]. This adaptive observer algorithm provides reliable estimates of the states (torque and angular velocity) of the system, of the friction coefficients related to the side forces $\left(\mu_{s}\right.$ and $\left.\mu_{k}\right)$ using the measurement of $\omega_{0}$.

\subsection{Observer equations}

In this section we recall the observer equations given in [14] as a copy of the plant equation plus some correction terms and then use them to derive an estimate of the tool face, $\hat{\phi}_{b}(t)$, as given in equation (24).
Let us denote with the $\hat{\cdot}$ superscript the estimated states and $e=\hat{\omega}_{0}-\omega_{0}$ the measured estimation error of the topdrive angular velocity. The observer equations given in [14] in terms of Riemann invariants read as follows

$$
\dot{\hat{\omega}}_{0}=a_{0}\left(\hat{\beta}_{p}(t, 0)-\hat{\omega}_{0}\right)+\frac{1}{I_{T D}} \tau_{m}-p_{0} e,
$$

$$
\begin{aligned}
\frac{\partial \hat{\alpha}_{i}}{\partial t}(t, x)+\left(c_{t}\right)_{i} \frac{\partial \hat{\alpha}_{i}}{\partial x}(t, x) & =\hat{\mathcal{S}}_{i}(t, x)-p_{\alpha}^{i}(x) e \\
\frac{\partial \hat{\beta}_{i}}{\partial t}(t, x)-\left(c_{t}\right)_{i} \frac{\partial \hat{\beta}_{i}}{\partial x}(t, x) & =\hat{\mathcal{S}}_{i}(t, x)-p_{\beta}^{i}(x) e
\end{aligned}
$$

where the index $i=c$ if we consider the collar section and $i=p$ if we consider the pipe section. The source term in each section are computed from the estimated states and friction factor

$$
\hat{\mathcal{S}}_{i}(t, x)=k_{t}\left(\hat{\alpha}_{i}(t, x)+\hat{\beta}_{i}(t, x)\right)+\frac{1}{J_{i} \rho} \hat{\mathcal{F}}_{i}(t, x),
$$

where $\hat{\mathcal{F}}$ has an expression analogous to $(4)$, the different variables being replaced by their estimates. Finally, the boundary conditions at the top and bottom, and between the drill string sections, are

$$
\begin{aligned}
\hat{\alpha}_{p}(t, 0) & =2 \hat{\omega}_{0}(t)-\hat{\beta}_{p}(t, 0)-P_{0} e \\
\hat{\beta}_{p}\left(t, L_{p}\right) & =\frac{\hat{\alpha}_{p}\left(t, L_{p}\right)(1-\bar{Z})+2 \bar{Z} \hat{\beta}_{c}\left(t, L_{p}\right)}{1+\bar{Z}}-P_{1} e \\
\hat{\alpha}_{c}\left(t, L_{p}\right) & =\frac{2 \bar{Z} \hat{\alpha}_{p}\left(t, L_{p}\right)-\hat{\beta}_{c}\left(t, L_{p}\right)(1-\bar{Z})}{1+\bar{Z}} \\
\hat{\beta}_{c}(t, L) & =\hat{\alpha}_{c}(t, L)
\end{aligned}
$$

where $\bar{Z}$ is defined as

$$
\bar{Z}=\left[\frac{c_{t}}{J G}\right]^{+} /\left[\frac{c_{t}}{J G}\right]^{-}
$$

The estimates of the friction factor are updated according to

$$
\begin{aligned}
& \dot{\hat{\mu}}_{s}(t)= \begin{cases}-l_{s} e, & \left|\hat{\omega}_{L_{c}}\right| \leq \omega_{c}, \\
0, & \left|\hat{\omega}_{L_{c}}\right|>\omega_{c},\end{cases} \\
& \dot{\hat{\mu}}_{k}(t)= \begin{cases}0, & \left|\hat{\omega}_{L_{c}}\right| \leq \omega_{c}, \\
l_{k} e, & \left|\hat{\omega}_{L_{c}}\right|>\omega_{c},\end{cases}
\end{aligned}
$$

Finally, the following saturation is used to improve robustness of the method: $\hat{\mu}_{s}=\max \left(\hat{\mu}_{s}, \hat{\mu}_{k}\right)$. The different constant and observer gains $a_{0}, p_{\alpha}^{i}, p_{\beta}^{i}, p_{0}, p_{1}, P_{0}, P_{1}, l_{s}, l_{k}$ are given in [14]. The initial condition of (13)-(20) can be arbitrarily chosen. This observer has been tested in [14] against field data where it was shown capable of providing a good estimation of the downhole velocity and of the sideforces friction parameters in the situation of an off-bottom bit. However, to estimate the tool face orientation, we need to combine it with the available (but delayed) orientation measurements. These measurements are available 


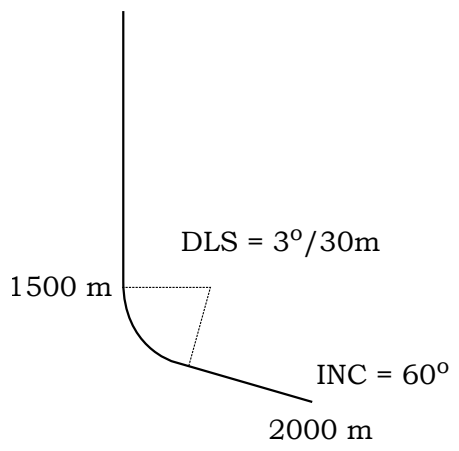

Figure 3: Wellbore survey for the simulation model. The latteral section is built with a $3^{\circ} / 30$ meter Dog-Leg Severity (DLS), which kicks off at 1500 meters Measured Depth.

every $T_{T}$ with a lag of $\tau_{T}$. Thus, our tool face estimation can only be corrected every $T_{T}$ seconds. More precisely, for any $k \geq 1$ and $k T_{T} \leq t<(k+1) T_{T}$, we consider the following estimator

$$
\begin{aligned}
\hat{\phi}_{b}(t)= & \int_{0}^{t} \frac{\hat{\alpha}(\nu, L)+\hat{\beta}(\nu, L)}{2} d \nu \\
& -\left(\hat{\phi}_{b}\left(k T_{T}-\tau_{T}\right)-\phi_{b}\left(k T_{T}-\tau_{T}\right)\right) .
\end{aligned}
$$

This estimation law is tested in simulations in the next section.

\subsection{Simulation results}

In this section, we show that the extended observer (13)(24) provides a reliable estimation of the tool face orientation in simulation. Note that we do not have for the moment any available measurements for the tool face orientation, making impossible any comparison with real field data. The simulation model we used is described in [8] with the wellbore survey shown in Figure 3, using the numerical implementation described in [8]. The kinetic friction is chosen to be equal to 0.28 , while the static friction is chosen to be equal to 0.45 which is similar to values reported using traditional friction tests in the field. The well represents a simple build used throughout the world. More discussion of this synthetic example, and reasoning for the choice of machine and system parameters, may be found in [7].

We consider a first scenario for which the top-drive velocity is tracking a reference trend (given in Figure 4, top) using a simple PI control law. We assume that the tool face orientation measurements are available every $T_{T}=10 \mathrm{~s}$ with a lag of $\tau_{T}=10 \mathrm{~s}$. We have pictured in Figure 4 (second subplot) the estimation of the friction coefficients which are given by our observer. The tool face orientation error, i.e. the difference between the real and estimated orientation in number of turns, is plotted in the third subplot of Figure 4. From these simulations, we can notice that the estimated orientation only converges toward the real one once the estimation of the friction terms provided by our observer becomes accurate enough. Moreover, any change in reference may create a transient during which the estimation of the friction terms is deteriorated, which in turn yields poor tool face estimates during this transcient (see Figure 4 for $t$ between 300s and 400s for instance). To avoid the deterioration of these friction estimates when the drill string is stationary, we propose the following calibration procedure: the driller sets a constant reference top drive velocity. This value is chosen large enough to guarantee enough excitation in the system (in particular, it is necessary that the torque breaks the static friction limitation). Once, the estimates of the friction parameters given by our observer have converged, we keep these values fixed. Thus, a change of reference won't imply new updates of the friction parameters estimation. This procedure is tested in simulations against the same scenario. We have pictured in Figure 4 the new estimation of the friction parameters (fourth subplot) and the tool face orientation error (last subplot). The accuracy of the orientation estimation is considerably improved compared to the previous case. The results are satisfactory for field applications, for which an estimation error of 0.25 turn for the orientation is acceptable. Note that as the friction parameters may slightly change with time, one can regularly repeat such a procedure to update the friction coefficients.

\section{Control of the tool face orientation}

In this section we consider the problem of the control of the tool face orientation. More precisely, given a final reference $\phi_{b}^{f}$, we want to design a reliable procedure to reach such a reference when we stop rotating.

\subsection{State of the art for tool face control}

Interest in closed loop control systems for directional drilling control has increased in the past three decades, particularly for well manufacturing scenarios - where many wells are drilled off the same well pad - and to reduce costs [17]. On current state-of-the-art land drilling rigs, tool face is predominantly controlled by a human directional driller interacting with a manual or or semi-automatic top drive control system. Some systems require the human driller to bump - or change - tool face through either angular position or angular velocity inputs to the top drive. The directional driller maintains a memory, or notepad, of previous bumps and the resulting change in tool face position, and reverts to these notes for subsequent bumps. More advanced systems implement some form of pipe rocking - an oscillation of top drive angular position to 'break' static friction along a portion of the horizontal drillpipe in sliding operations - and thus bundle the tool face bumping procedure as part of a semi-automated rocking routine [18]. Several feedback control systems have been tested in the field for closed loop tool face control, but have struggled to outperform human drillers, and thus remain in limited deployment and results remain unpublished. The root cause often reported is the latency of 

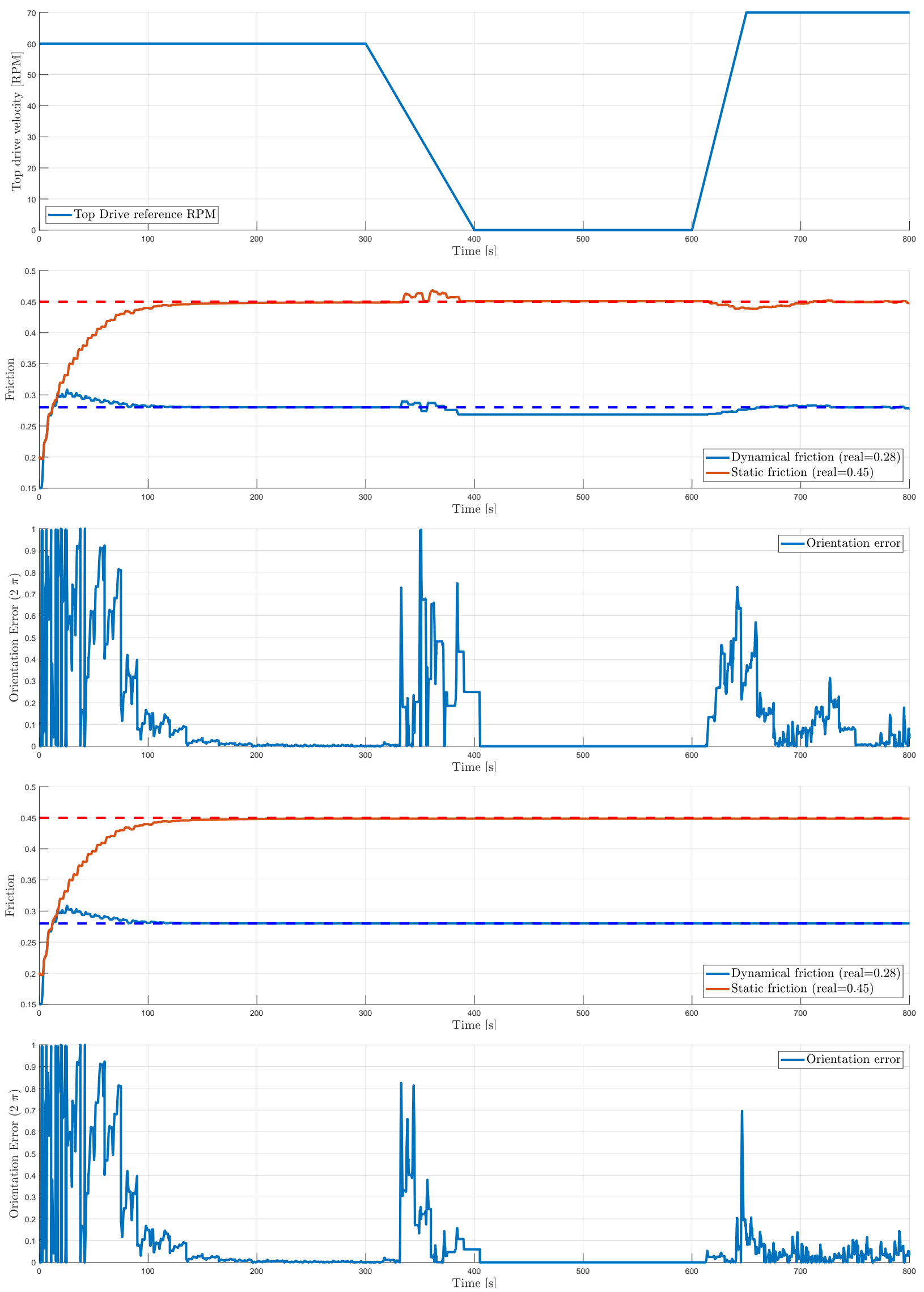

Figure 4: Top drive velocity (top), estimated friction coefficients (second), orientation error (third) using the observer (13)-(24), estimated friction coefficients when the estimation is stopped when $t \geq 200$ s (fourth) and orientation error when the estimation is stopped when $t \geq 200$ s (bottom) 
tool face measurement and the non-linear nature of static and kinematic friction. Moreover, a crucial point is that one cannot directly act on the state $\phi_{b}$, but only on its derivative $\omega(t, L)$. In the following sections we recall the existing results regarding the control of Bottom Hole Assembly (BHA) velocity, before presenting three potential closed loop control strategies for the tool face orientation.

\subsection{Feedback controllers for the BHA velocity}

As we can only act on the orientation by modifying the BHA velocity (using a top-drive actuation), it might be of interest to consider feedback laws that have been designed to control BHA velocity while avoiding the effects of stickslip [19, 20]. For a majority of drilling rigs in the field, the actuation is done through $\mathrm{AC}$ electric top drives, using a variety of variable frequency drives which are capable of highly accurate, high frequency, rotary speed control. Two types of stick-slip mitigation controllers have been widely deployed: the older SoftTorque-SoftSpeed systems and the newer ZTorque systems. These control laws have been proved to be more efficient compared to simple stiff PI controller [20]. Such control laws have been improved in [19] by completing them by a feed-forward action that does not disturb their closed-loop behavior. Due to its simplicity, the industry standard controller that is most often used is a high gain PI control to ensure rapid tracking of the top drive set point [21]. The control signal has the following structure

$$
\tau_{m}=-\left(C \star\left(\omega_{S P}-\omega_{0}\right)\right),
$$

where $\omega_{S P}$ is the set point for the top drive velocity and where the controller impulse response $C(t)$ is composed of a proportional term and of an integral term as described in (10). This kind of controllers have been improved to handle and compensate the effect of torsional vibrations and can be used to stabilize the downhole velocity around the same set point $\omega_{S P}$.

\subsubsection{SoftTorque and ZTorque}

The current industry standard in handling torsional vibrations are the two products NOV's SoftSpeed [22, 23] and Shell's SoftTorque [24, 25]. The objective of these solutions is to reduce the reflection coefficient at the top drive in a certain key frequency range [21]. Some improvements for this stick-slip mitigation control have been done by Shell in ZTorque. In this new solution, the reflection coefficient of the top drive is minimized for a wider range of frequencies by measuring the torque between the drill string and top-drive. The feedback control law is used to "artificially" have the top-drive match the impedance of the drill-pipe, $\zeta_{p}=J_{p} \sqrt{G_{p} \rho}$. The block diagram of the Z-torque control law is given in Fig 5 .

It has been shown in [20] that the Z-torque control law effectively removes stick-slip oscillations at the costs of delivering high instantaneous torque to the top drive

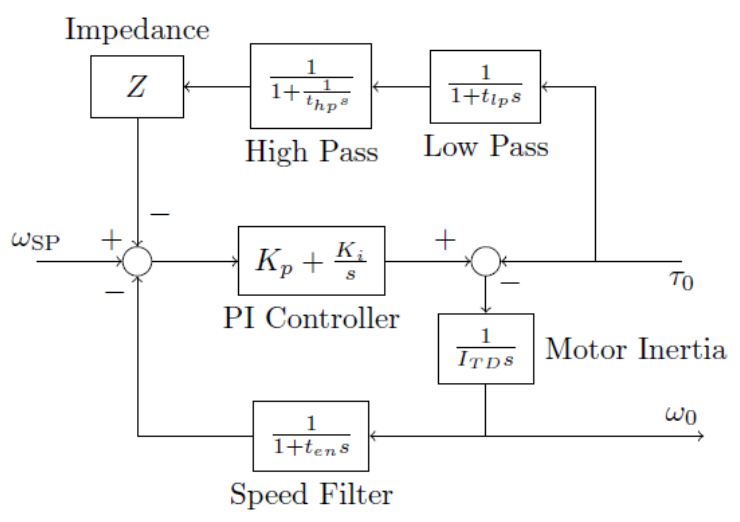

Figure 5: Control diagram for a ZTorque system with direct pipe torque measurement. For ZTorque $Z=\frac{1}{\zeta_{p}}$ is used. If $Z=$ 0 , the control diagram is equivalent to a SoftTorque or stiff speed controller system.

to allow for impedance matching through rapid velocity changes and a significantly slower response in rotational velocity of the top-drive. Z-torque also requires extra instrumentation, namely a measurement of the torque acting from the drill string on the top-drive, to work effectively.

\subsubsection{Feed-forward trajectory design}

A strategy to avoid torsional stick-slip oscillations (especially at the start-up of a drilling operation) has been developed in [19]. It consists in a feed-forward controller, that can be added to the standard industry feedback controllers. This feed-forward control law can be used to track any downhole velocity profile. The control signal is now composed of three terms

$$
\tau_{m}=u_{c}+u_{f}+u_{d},
$$

where $u_{c}(t)=-\left(C \star\left(\omega_{S P}-\omega_{0}\right)\right)(t)$ is a feedback term, $u_{f}$ is a feed-froward term that ensures tracking and $u_{d}$ corresponds to a compensation of the friction term (which is modeled as a disturbance). This conforms to a canonical 3DOF controller architecture [12]. Note that the disturbance feed-forward term is needed since the disturbance canceling imposes a trajectory on the top-drive velocity. More precisely, this controller exploits the differential flatness of the model $[26,27]$, which means that the control input can be parametrized as a function of one output (here the BHA velocity $\omega(t, L)$ ). Such a control law has been tested in a extensive simulation study in [19] with the field data validated simulation model [7].

\subsection{Tool face orientation control procedures}

In this section, we describe three procedures to control the tool face orientation. These procedures are inspired by what is currently done by human drillers, but exploit the improved precision and data utilization enabled by automatic control. These three procedures vary in terms of requirements on available instrumentation and model accuracy and in terms of time needed to be completed: 
1. Feed-forward control: This procedure starts with stable rotation off-bottom and assumes the availability of an estimate of current tool face orientation. Using the flatness of the drilling system, an admissible trajectory is generated that controls the tool face orientation to the desired value at the same time as bitrotation stops. The flatness property of the model [19] is used to obtain the required actuation and measurement trends which are then tracked by the $3-\mathrm{DOF}$ controller described in Section 4.2.2.

- Requirements: Stable off-bottom rotation, good model of the drill string and correct estimate of tool face orientation while rotating.

- Performance: One iteration: Correct orientation immediately when stopping.

2. Rotating iteration: In this procedure we again require stable rotation off-bottom, but use no assumption on model correctness. During rotation off-bottom we smoothly change the reference to zero to stop bit rotation. As the delayed measurement of the stationary tool face orientation is obtained, a heuristic relationship between the setpoint change and tool face orientation can be established, and desired orientation is achieved by iterating the procedure.

- Requirements: Stable off-bottom rotation, correct estimate of tool face orientation while rotating.

- Performance: Two iterations.

3. Bump iteration: This procedure rotates the topdrive up to the release torque, and the top-drive velocity reference is then changed by a bump. This bump induces a change of orientation for the tool face. Iterating, with changes in the amplitude of the bump, we estimate the parameters of an affine relation between the bump amplitude and the corresponding change in tool face orientation. After two iterations, the correct amplitude can be computed and the correct tool face achieved on the third iteration.

- Requirements: None.

- Performance: Three iterations.

The performance and requirements of these three different procedures are summarized in Table 6 .

\subsubsection{Feed-forward Procedure}

As explained in [19], a feed-forward control law can be added to the standard industry feedback controllers to track any downhole velocity profile. These trajectory profiles are usually constructed using a mollifier (semianalytical function), which guarantees that the transition trajectories are booth smooth and have vanishing derivative at the end and start point (see [19] for details). This procedure requires steady rotation off-bottom. For many wells this requires the use of Z-torque or other feedback control (or the startup procedure described in [19]) to achieve. In the present simulation study, we assume the use of a rig with the Z-torque control. Once this steady state is reached (at time $T_{0}$ ), we estimate the current orientation using our observer. We are able to define a reference trajectory to go from this current tool face orientation to the final desired one (eventually adding it $2 k \pi$ to have a realistic smooth trajectory). From this trajectory, we can generate the corresponding BHA velocity trajectory. Using the flatness property of the model, we obtain the control input that tracks this trajectory. We finally use the feed-forward control law (26) to reach the desired orientation. This procedure is described in Algorithm 1. It has been tested in simulation against the model described in [8] with the wellbore survey shown in Figure 3. The kinetic and static friction coefficients are still chosen as 0.28 and 0.45 , respectively, which is similar to field scenarios. The desired orientation is set to $\frac{\pi}{4}$ radians $(=0.125$ turn or $\left.45^{\circ}\right)$. The actuation is subject to a maximum torque saturation of $30 \mathrm{kNm}$. In this scenario, we assume that the friction parameters are known (or have been estimated using the procedure described in Section 3). The different results are pictured in Figure 7.

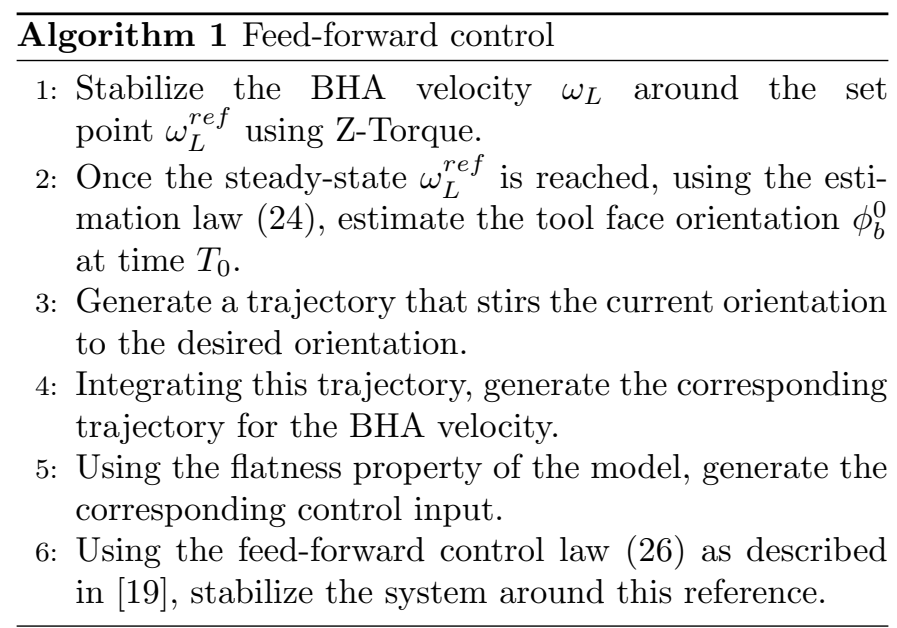

\subsubsection{Rotating iteration}

The idea of this procedure consists in stirring the system into the same initial state, for which the BHA velocity is constant. Hence, stable rotation is assumed. Once this steady state has been reached, we smoothly change the velocity reference to zero. Using our observer and the available measurements, we can then estimate the variation of orientation induced by the change of reference. Based on the obtained result, we can adjust the procedure accordingly, anticipating the variation of orientation induced by the change of reference and stopping the actuation earlier (using the estimation of the orientation given by our observer).

The desired orientation will be denoted $\phi_{b}^{f}$, while the constant BHA velocity set point will be denoted $\omega_{T D}^{r e f}=$ $\omega_{L}^{r e f}$ (for instance $\omega_{L}^{r e f}=25 R P M$ ). This set point has 


\begin{tabular}{|c|c|c|}
\hline Controller & Requirements & Performance \\
\hline Feed-forward control & $\begin{array}{c}\text { Good model of drill string } \\
\text { Stable off-bottom rotation } \\
\text { Tool face orientation estimation }\end{array}$ & One iteration \\
\hline Rotating iteration & $\begin{array}{c}\text { Stable off-bottom rotation } \\
\text { Tool face orientation estimation }\end{array}$ & Two iterations \\
\hline Bump iteration & Estimation of the release torque & Three iterations \\
\hline
\end{tabular}

Figure 6: Comparison of the requirements and performance for the three proposed procedures

to be large enough to break the static friction and initiate rotation [7]. The complete procedure is described in Algorithm 2.

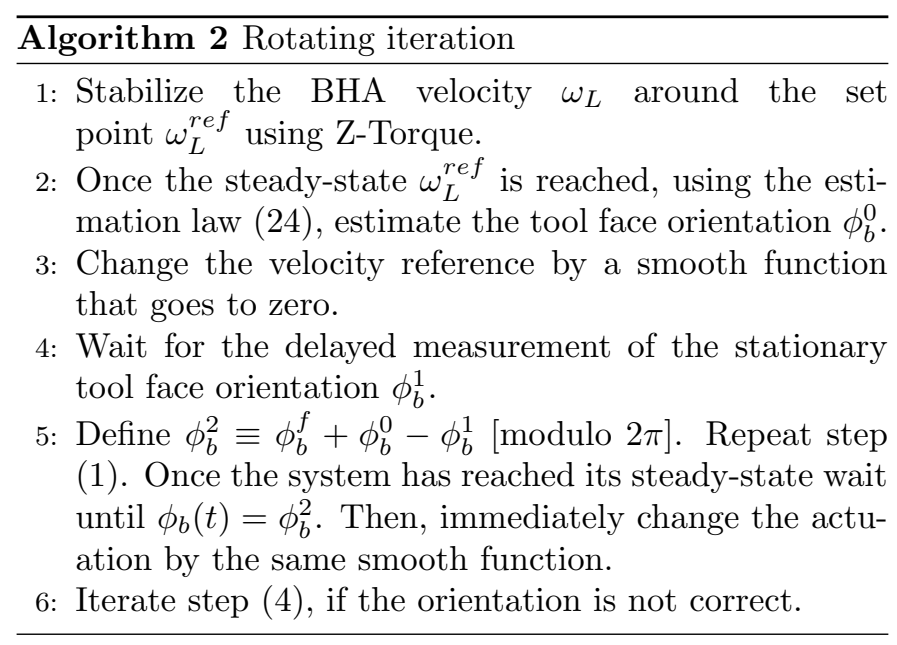

Note that the quantity $\phi_{b}^{2} \equiv \phi_{b}^{f}+\phi_{b}^{0}-\phi_{b}^{1}$ [modulo $2 \pi$ ] corresponds to the difference between the desired orientation and the variation of orientation induced by the change of reference. Such a procedure has been tested in simulation against the model described in [8] with the wellbore survey shown in Figure 3. The kinetic and static friction coefficients are still chosen as 0.28 and 0.45 , respectively, which is similar to field scenarios. The desired orientation is set to $\frac{\pi}{4}$ radians $\left(=0.125\right.$ turn or $\left.45^{\circ}\right)$. The actuation is subject to a maximum torque saturation of $30 \mathrm{kNm}$. We have pictured in Fig. 8 the evolution of the BHA and top drive angular velocity, of the top drive torque and of the tool face orientation.

In this ideal situation (no delay, or noise), Algorithm 2 leads to excellent results. The main drawback of such a procedure is that it requires to know the orientation at each time (to know when the actuation has to be stopped). Thus, the performance is directly related to the accuracy of the observer estimates.

\subsubsection{Bump iteration}

This last procedure relies on the fact that the BHA can only start rotating once there is enough energy in the drill string. This means that the motor torque has reached a critical value (which corresponds to the static torque imposed by the side forces), known as the release torque. The proposed approach consists in stirring our system into this known repeatable state (for which the motor torque has almost reached its release value) and then setting a bump as the new top-drive velocity set point. Due to (4), once the release torque is reached, we have a linear relation between the amplitude of the bump which is sent and the corresponding change of tool face orientation. This is due to the fact that when the drill string breaks free from the static friction, the system dynamics are linear (the kinematic friction term is linear). We can then measure the change of orientation induced by the bump. Using the linearity, we can adjust the bump amplitude and repeat the procedure. More precisely, let us consider a stationary drill string for which we can measure the tool face orientation $\phi_{b}^{1-}$ using the available sensor. We then stabilize the top-drive velocity around a given small reference (namely $15 \mathrm{RPM}$ ). The torque in the drill string progressively increases. Once the top-drive torque (which can be directly measured) almost reaches its release value, we change the constant top-drive velocity reference by a bump with a given amplitude $A_{1}$ (that has to be large enough). Due to this bump, as the release torque is reached, the drill bit starts rotating for a short period of time. After the operation, we can measure the resulting tool face orientation $\phi_{b}^{1+}$ and compute the variation of orientation induced by the bump $(\Delta \phi)_{1}=\phi_{b}^{1+}-\phi_{b}^{1-}$. We then iterate the procedure adjusting the amplitudes of the bump and using the linear relation between the amplitude of the bump and the change of orientation induced by this bump. As described below, after several bumps, the bit orientation reaches its desired value. The first part of the procedure is necessary to guarantee the same initial condition before sending the bump (just before the release torque). This requires the knowledge of the value of the release torque. This value can be estimated using the method presented in [19] (that however requires the estimation on the friction terms, which is possible using our observer). The friction terms will be assumed known here. Finally it is worth mentioning that this procedure does not require to estimate the orientation as we only have to wait until the required measurements become available. The complete procedure is summarized in Algorithm 3.

This procedure has been tested against the simulation model described in Section 4.3.2 (with a torque saturation of $30 \mathrm{kNm}$ ). For this model the release torque is equal 

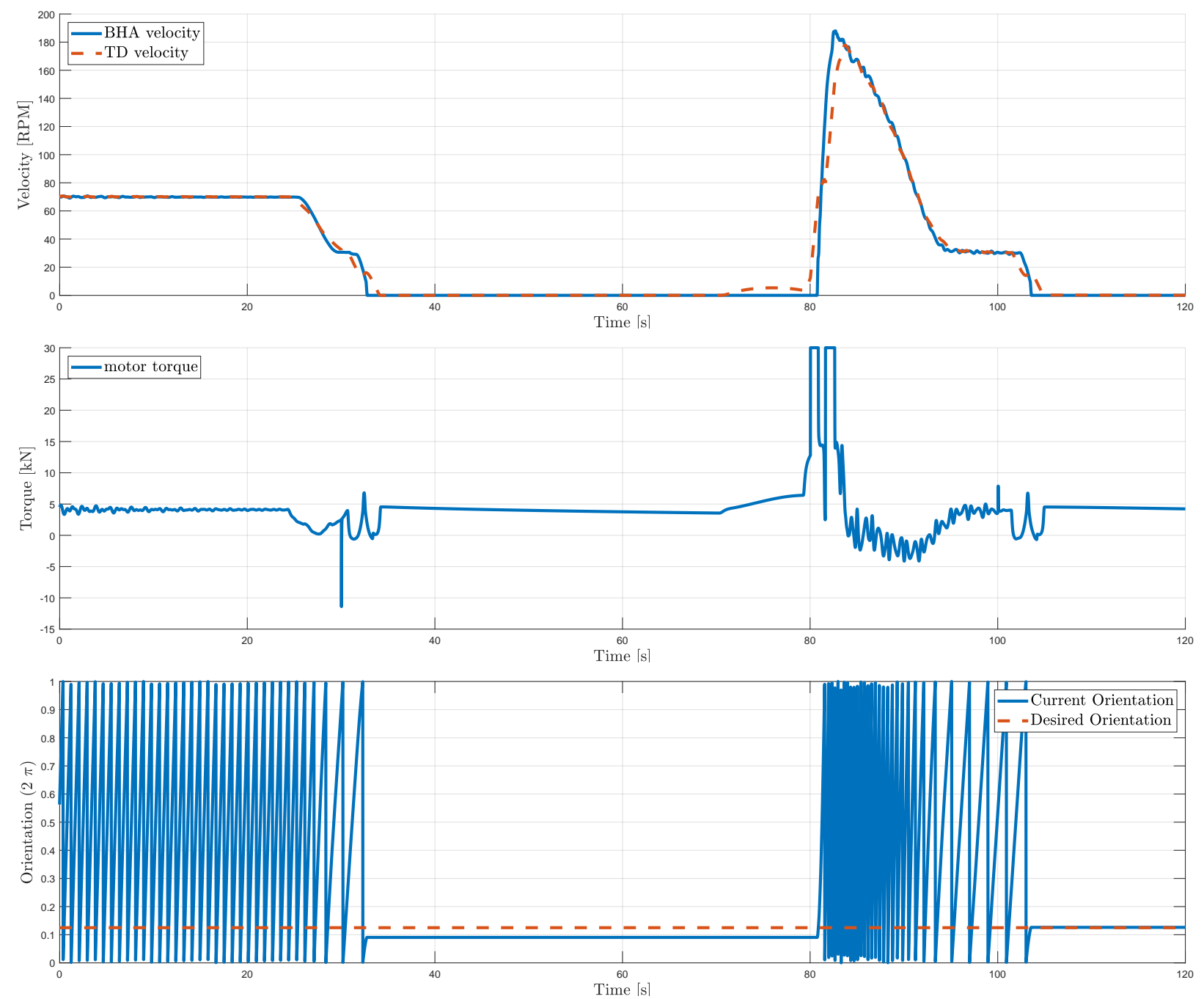

Figure 7: Time evolution of the top drive and BHA velocity (top), of the torque (middle) and the orientation, expressed in turns (bottom) using the feed-forward flatness control of the BHA orientation (Algorithm 1). The control is shown both from a steady orientation and from a stationary condition. The desired orientation is equal to $\frac{\pi}{4}$ radians $(=0.125$ turn).

to $17 \mathrm{kNm}$. We choose the top-drive reference velocity to be equal to 15 RPM, while the bit-orientation we want to reach is equal to $\frac{\pi}{4}$ radians $(=0.125$ turn). In Table 2 , we have shown the variations of orientation $(\Delta \phi)_{i}$ induced by different bumps. The bumps have the same duration, only their amplitudes $A_{i}$ begin different. The amplitudes are chosen such that the difference $\left(d_{A}\right)_{i}=A_{i}-A_{i-1}$ remain constant. The relative variation of orientation after each bump is expressed in terms of turn, instead of radians (which explain why the different values are all in the interval $[0,1))$.

Using the results given in Table 2 , it is possible to model $(\Delta \phi)_{i}$ as an affine function of $A_{i}$. We obtain the following linear regression

$$
\begin{aligned}
(\Delta \phi)_{i} & =0.26\left(A_{i}-5.2\right)+0.79[\text { modulo } 1] \\
& =\bar{\Delta}\left(A_{i}-A_{1}\right)+(\Delta \phi)_{1}[\text { modulo } 1],
\end{aligned}
$$

where $\bar{\Delta}$ is the slope of the regression.

\begin{tabular}{|c|c|c|c|}
\hline Iteration & $A_{i}$ & $(\Delta \phi)_{i}$ & $(\Delta \phi)_{i}-(\Delta \phi)_{i-1}$ \\
\hline 1 & 5.2 & 0.79 & N.A \\
2 & 5.3 & 0.823 & +0.024 \\
3 & 5.4 & 0.849 & +0.026 \\
4 & 5.5 & 0.875 & +0.026 \\
5 & 5.6 & 0.901 & +0.026 \\
6 & 5.7 & 0.928 & +0.027 \\
\hline
\end{tabular}

Table 2: Computation of $(\Delta \phi)_{i}$ and $(\Delta \phi)_{i}-(\Delta \phi)_{i-1}$ (expressed in turns) for 6 different bumps (whose amplitudes are characterized by $A_{i}$ ) 

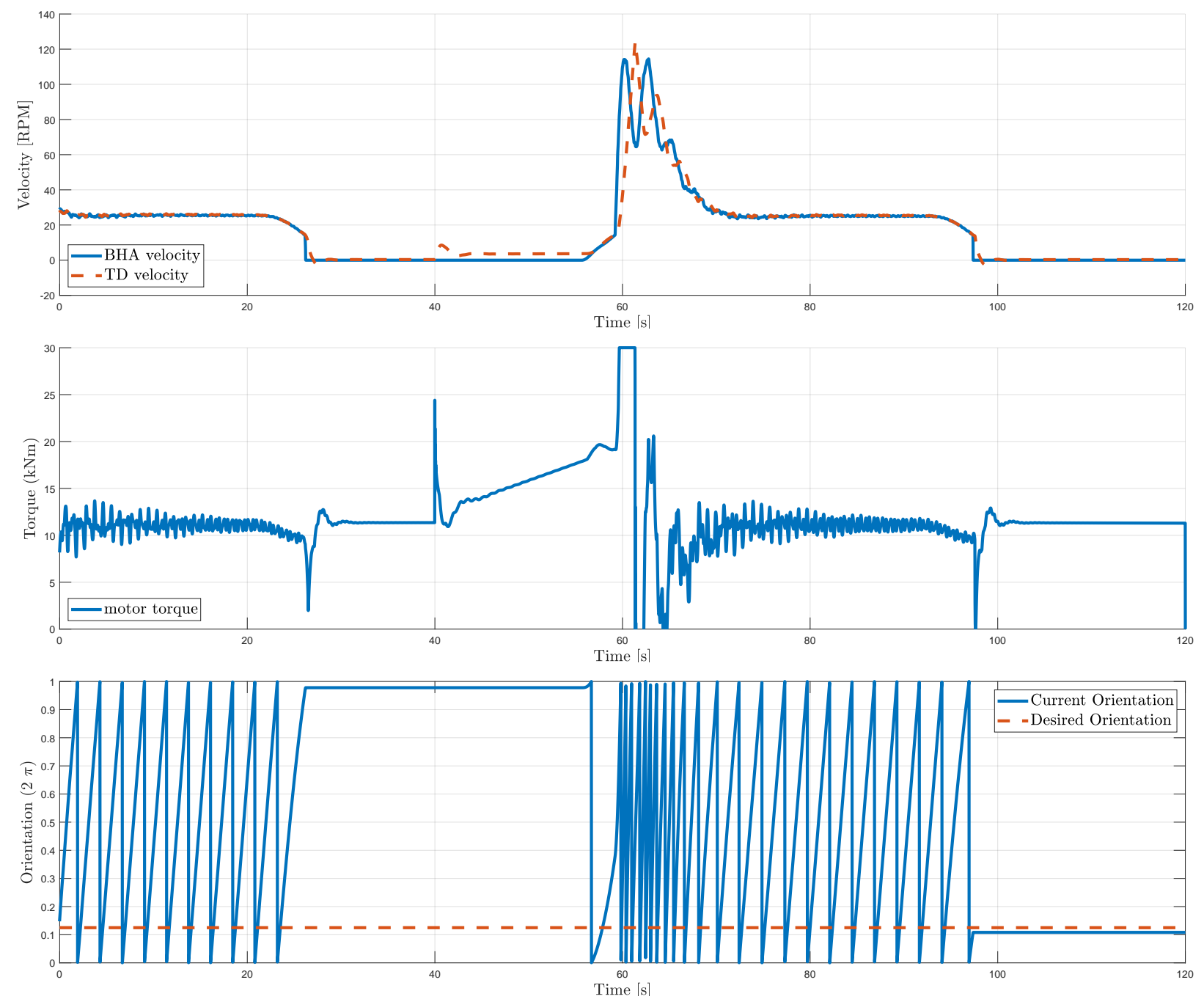

Figure 8: Time evolution of the top drive and BHA velocity (top), of the torque (middle) and the orientation, expressed in turns (bottom) following Algorithm 2. The desired orientation is equal to $\frac{\pi}{4}$ radians ( $=0.125$ turn).

However convenient this linear expression may appear at first sight, one must be aware that, due to the fact that we are only able to measure the relative variation of the tool face orientation, it can only be used to compute the relative variation of orientation when $A_{i}=A_{0}+n\left(d_{A}\right)(n$ being an integer). Let us give an example to illustrate this problem. If a variation of amplitude of $d_{A}$ implies a relative change of orientation of 0.2 turns, then a variation of amplitude of $10 d_{A}$ would imply a relative change of orientation of 2 turns, which will be seen as 0 (no relative displacement). Thus, knowing only the relative change of orientation for a variation of amplitude of $10 d_{A}$ it becomes impossible to guess a relative change of orientation induced by a change of amplitude of $d_{A}$. This limitation implies that the desirable orientation cannot be perfectly reached. Thus, $d_{A}$ has to be chosen small enough to guarantee an acceptable precision. Obviously, if the variation of orientation is known absolutely (in number of turns) and not relatively, this problem disappears. This can be achieved by using the orientation estimation provided by (24).

Being aware of this intrinsic limitation, we can however use expression (27) to obtain the amplitude of the future bump we have to send. In the considered example, we initiate the procedure with two bumps for which $A_{1}=5.2, A_{2}=5.3$. Before sending the third bump, we measure $\phi_{b}^{3-}$. As we want $\phi_{b}^{3+}=0.125$ we can compute the corresponding orientation variation $(\Delta \phi)_{3}$ we need to generate. Using (27), we obtain the corresponding bump amplitude $A_{3}$ (here we have chosen $A_{3}=4$ ). We have pictured in Figure 9 the time evolution of the topdrive and BHA velocity, motor torque and tool face orientation for this example. Note that a torque saturation of $30 \mathrm{kNm}$ has been used in these simulations. For this example only three bumps were required.

The whole procedure can be summarized by Algorithm 3 . 

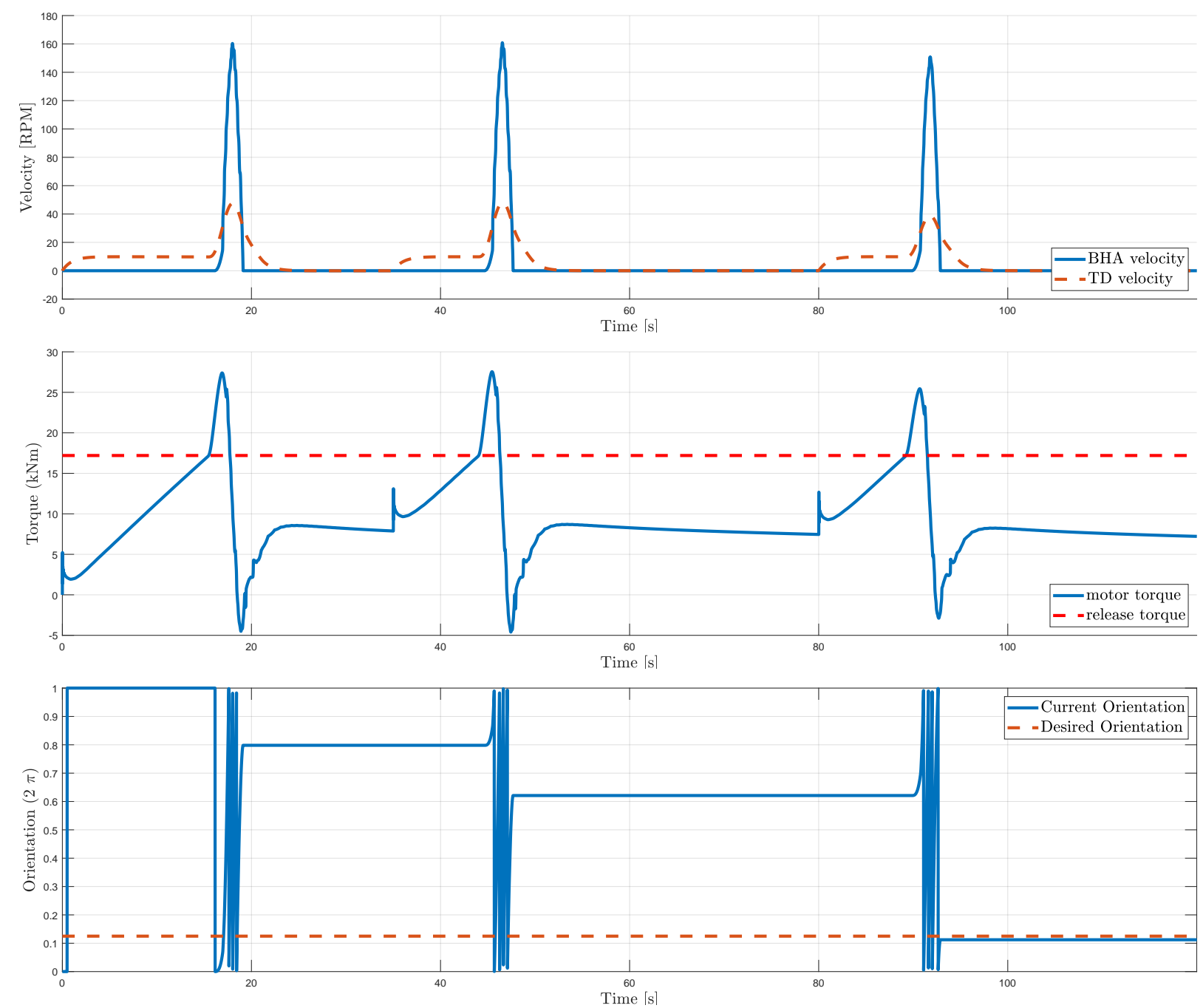

Figure 9: Time evolution of the top drive and BHA velocity (top), of the torque (middle) and the orientation, expressed in turns (bottom) following Algorithm 3. The desired orientation is equal to $\frac{\pi}{4}$ radians ( $=0.125$ turn).

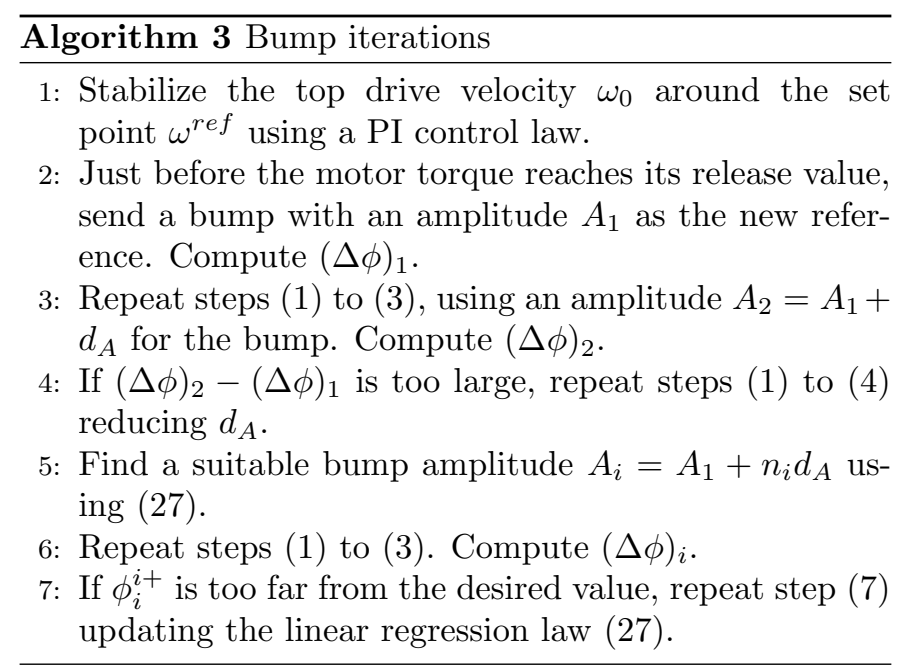

\section{Conclusion}

In this paper we have proposed a model and an algorithm to estimate drill bit velocity and orientation position - the tool face - while drilling directional wells. The proposed algorithm only uses surface measurements and sampled and delayed tool face orientation measurements. The approach relies on an observer combined with an update law of the static and kinematic friction factors used in a non-linear Coulomb friction model and an update for the estimation of the orientation based on the available measurements. Such an observer has been successfully used on synthetic data. Combining this observer with existing stick-slip mitigation feedback laws, we have derived three procedures that can be used to control the drill bit orientation. These procedures have been successfully used in simulation using a field validated torsional drill string simulator and demonstrate rapid, accurate and robust setting of tool face. This presents a new advance towards effective and efficient closed loop directional drilling control. 


\section{Acknowledgments}

Support for this work came from Canada's Natural Sciences and Engineering Research Council's Collaborative Research and Development Grant \# CRDPJ 530374-18 in collaboration with Precision Drilling Corporation. Additional support came from the University of Calgary's Canada First Research Excellence Fund Program, the Global Research Initiative in Sustainable Low Carbon Unconventional Resources and by the European Union's Seventh Framework Programme for research, technological development and demonstration under Marie Curie grant agreement no [608695], through FRIPRO Mobility Grant Fellowship Programme (FRICON).

This work was also supported by the University of Calgary's Canada First Research Excellence Fund Program, the Global Research Initiative in Sustainable Low Carbon Unconventional Resources.

\section{References}

[1] D Stroud, M Russell, S Peach, et al. Development of the industry's first slimhole point-the-bit rotary steerable system. In SPE Annual Technical Conference and Exhibition. Society of Petroleum Engineers, 2003.

[2] S. Schaaf, C.R. Mallary, D. Pafitis, et al. Point-the-bit rotary steerable system: Theory and field results. In SPE Annual Technical Conference and Exhibition. Society of Petroleum Engineers, 2000.

[3] J. Clegg, C. Mejia, S. Farley, et al. A paradigm in rotary steerable drilling-market demands drive a new solution. In SPE/IADC International Drilling Conference and Exhibition. Society of Petroleum Engineers, 2019.

[4] L. W. Ledgerwood III, R. W. Spencer, O. Matthews, J. AR. Bomidi, J. Mendoza, J. M. Hanson, et al. The effect of bit type on reactive torque and consequent tool-face-control anomalies. SPE Drilling \&G Completion, 31(02):95-105, 2016.

[5] J. F. Inyang, I. J.and Whidborne and M. T. Bayliss. Directional drilling attitude control with input disturbances and feedback delay. IFAC-PapersOnLine, 50(1):1409-1414, 2017.

[6] P. Weijermans, J. Ruszka, H. Jamshidian, M. Matheson, et al. Drilling with rotary steerable system reduces wellbore tortuosity. In SPE/IADC drilling conference. Society of Petroleum Engineers, 2001.

[7] U. J. Aarsnes and R. J. Shor. Torsional vibrations with bit off bottom: Modeling, characterization and field data validation. Journal of Petroleum Science and Engineering, 163:712-721, apr 2018.

[8] U. J. F. Aarsnes and N. van de Wouw. Axial and torsional self-excited vibrations of a distributed drill-string. Journal of Sound and Vibration, 444(March):127-151, 2019.

[9] C. Germay, V. Denoël, and E. Detournay. Multiple mode analysis of the self-excited vibrations of rotary drilling systems. Journal of Sound and Vibration, 325(1-2):362-381, aug 2009.

[10] M C Sheppard, C Wick, and T Burgess. Designing Well Paths To Reduce Drag and Torque. SPE Drilling Engineering, 2(04):344-350, dec 1987.

[11] U. J. F. Aarsnes and O. M. Aamo. Linear stability analysis of self-excited vibrations in drilling using an infinite dimensional model. Journal of Sound and Vibration, 360:239-259, jan 2016.

[12] K. J. Åström and R. M. Murray. Feedback systems: an introduction for scientists and engineers. Princeton university press, 2nd edition, 2010.

[13] R. J. LeVeque. Finite volume methods for hyperbolic problems. Cambridge University Press, 2002.
[14] U. J. F. Aarsnes, J. Auriol, F. Di Meglio, and R. J. Shor. Estimating friction factors while drilling. Journal of Petroleum Science and Engineering, 2019.

[15] I. N. de Almeida Jr, P. D. Antunes, F. O. C. Gonzalez, R. A. Yamachita, A. Nascimento, and J. L. Goncalves. A review of telemetry data transmission in unconventional petroleum environments focused on information density and reliability. Journal of Software Engineering and Applications, 8(09):455, 2015.

[16] F. Di Meglio, P.-O. Lamare, and U. J. F. Aarsnes. Robust output feedback stabilization of an ODE-PDE-ODE interconnection. Submitted, (October):1-11, 2018.

[17] T. Jacobs et al. Automated drilling technologies showing promise. Journal of Petroleum Technology, 67(06):50-55, 2015.

[18] J. K. Wilson, G. Heisig, et al. Investigating the benefits of induced vibrations in unconventional horizontals via nonlinear drill string dynamics modeling. In SPE/IADC Drilling Conference and Exhibition. Society of Petroleum Engineers, 2015.

[19] U. J. F Aarsnes, F. Di Meglio, and R. J. Shor. Avoiding stick slip vibrations in drilling through startup trajectory design. Journal of Process Control, 70:24-35, oct 2018.

[20] U. J. F. Aarsnes, F. Meglio, and R. J. Shor. Benchmarking of Industrial Stick-Slip Mitigation Controllers. In IFAC Workshop on Automatic Control in Offshore Oil and Gas Production, 2018.

[21] A. Kyllingstad. A Comparison of Stick-Slip Mitigation Tools. In SPE/IADC Drilling Conference and Exhibition, number March, pages 14-16. Society of Petroleum Engineers, mar 2017.

[22] G. W. Halsey, A. Kyllingstad, and A. Kylling. Torque Feedback Used to Cure Slip-Stick Motion. In SPE Annual Technical Conference and Exhibition, pages 277-282. Society of Petroleum Engineers, apr 1988.

[23] Å. Kyllingstad and P. J. Nessjøen. A New Stick-Slip Prevention System. In Proceedings of SPE/IADC Drilling Conference and Exhibition, number March, pages 17-19, 2009.

[24] S. Dwars. Recent Advances in Soft Torque Rotary Systems. In Proceedings of 2015 SPE/IADC Drilling Conference, number March, pages 17-19, 2015.

[25] D. J. Runia, S. Dwars, and I. P. J. M. Stulemeijer. A brief history of the Shell "Soft Torque Rotary System" and some recent case studies. In SPE/IADC Drilling Conference, pages 69-76. Society of Petroleum Engineers, mar 2013.

[26] M. Fliess, J. Lévine, P. Martin, and P. Rouchon. Flatness and defect of non-linear systems: introductory theory and examples. International journal of control, 61(6):1327-1361, 1995.

[27] H. Mounier, J. Rudolph, M. Fliess, and P. Rouchon. Tracking control of a vibrating string with an interior mass viewed as delay system. ESAIM: Control, Optimisation and Calculus of Variations, 3:315-321, 1998. 